\title{
On the existence of solutions for nonhomogeneous Schrödinger-Poisson system
}

\author{
Lixia Wang ${ }^{1}$, Shiwang $\mathrm{Ma}^{2}$ and Xiaoming Wang ${ }^{3 *}$
}

\author{
"Correspondence: \\ wxmsuda03@163.com \\ ${ }^{3}$ School of Mathematics and \\ Computer Science, Shangrao \\ Normal University, Shangrao, Jiangxi \\ 334001, China \\ Full list of author information is \\ available at the end of the article
}

\begin{abstract}
In this paper, we study the existence of solutions for the following nonhomogeneous Schrödinger-Poisson systems:

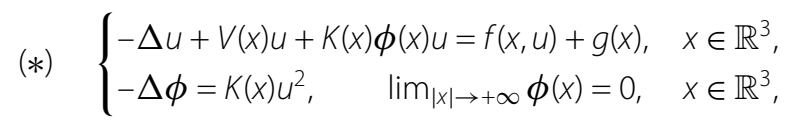

where $f(x, u)$ is either sublinear in $u$ as $|u| \rightarrow \infty$ or a combination of concave and convex terms. Under some suitable assumptions, the existence of solutions is proved by using critical point theory.
\end{abstract}

MSC: 35B33; 35J65; 35Q55

Keywords: Schrödinger-Poisson systems; sublinear nonlinearities; concave and convex nonlinearities; variational methods

\section{Introduction}

The following Schrödinger-Poisson system:

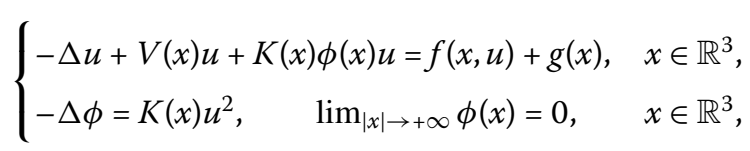

arises in several interesting physical contexts. It is well known that (1.1) has a strong physical meaning since it appears in quantum mechanical models (see $[1,2]$ ) and in semiconductor theory (see [3-6]). From the point view of quantum mechanics, the system (1.1) describes the mutual interactions of many particles [7]. Indeed, if the terms $f(x, u)$ and $g(x)$ are replaced with 0 , then problem (1.1) becomes the Schrödinger-Poisson system. In some recent work (see [8-16]), different nonlinearities are added to the Schrödinger-Poisson equation, giving rise to the so-called nonlinear Schrödinger-Poisson system. These nonlinear terms have been traditionally used in the Schrödinger equation to model the interaction among particles.

Many mathematicians have devoted their efforts to the study of (1.1) with various nonlinearities $f(x, u)$. We recall some of them as follows.

(c) 2016 Wang et al. This article is distributed under the terms of the Creative Commons Attribution 4.0 International License (http://creativecommons.org/licenses/by/4.0/), which permits unrestricted use, distribution, and reproduction in any medium, provided you give appropriate credit to the original author(s) and the source, provide a link to the Creative Commons license, and indicate if changes were made. 
The case of $g \equiv 0$, that is, the homogeneous case, has been studied widely in $[6,8,11,17$ 25]. In 2010, Cerami and Vaira [10] study system (1.1) in the case of $f(x, u)=a(x)|u|^{p-2} u$ with $4<p<6$ and $a(x)>0$. In order to recover the compactness of the embedding of $H^{1}\left(\mathbb{R}^{3}\right)$ into the Lebesgue space $L^{s}\left(\mathbb{R}^{3}\right), s \in[2,6)$, they establish a global compactness lemma. They prove the existence of positive ground state and bound state solutions without requiring any symmetry property on $a(x)$ and $K(x)$.

In 2012, Sun et al. [26] consider a more general case, that is, $f(x, u)=a(x) \tilde{f}(u)$ where $\tilde{f}$ is asymptotically linear at infinity, i.e. $\tilde{f}(s) / s \rightarrow c$ as $s \rightarrow+\infty$ with a suitable constant $c$. They establish a compactness lemma different from that in [10] and prove the existence of ground state solutions. In [27], Ye and Tang study the existence and multiplicity of solutions for homogeneous system of (1.1) when the potential $V$ may change sign and the nonlinear term $f$ is superlinear or sublinear in $u$ as $|u| \rightarrow \infty$. For the Schrödinger-Poisson system with sign-changing potential, see [14, 28].

Huang et al. [12] study the case that $f(x, u)$ is a combination of a superlinear term and a linear term. More precisely, $f(x, u)=k_{1}(x)|u|^{p-2} u+\mu h_{1}(x) u$, where $4<p<6$ and $\mu>0$, $k_{1} \in C\left(\mathbb{R}^{3}\right), k_{1}$ changes sign in $\mathbb{R}^{3}$ and $\lim _{|x| \rightarrow+\infty} k_{1}(x)=k_{\infty}<0$. They prove the existence of at least two positive solutions in the case that $\mu>\mu_{1}$ and near $\mu_{1}$, where $\mu_{1}$ is the first eigenvalue of $-\Delta+$ id in $H^{1}\left(\mathbb{R}^{3}\right)$ and with weight function $h_{1}$. In $[29,30]$, the authors consider the critical case of $p=6$; in [31] one studies the case of $p=4$.

Sun et al. [32] get infinitely many solutions for (1.1), where we have the nonlinearity $f(x, u)=k_{2}(x)|u|^{q-2} u-h_{2}(x)|u|^{l-2} u, 1<q<2<l<\infty$, i.e. the nonlinearity involving a combination of concave and convex terms. For more results on the effect of concave and convex terms of elliptic equations, see $[33,34]$ and the references therein.

Next, we consider the nonhomogeneous case of (1.1), that is, $g \not \equiv 0$. The existence of radially symmetric solutions is obtained for above nonhomogeneous system in [23]. Chen and Tang [35] obtain two solutions for the nonhomogeneous system with $f(x, u)$ satisfying Amborosetti-Rabinowitz type condition and $V$ being non-radially symmetric. In [36, 37], the system with asymptotically linear and 3-linear nonlinearity is considered. For more results on the nonhomogeneous case, see $[38,39]$ and the references therein.

Motivated by the work mentioned above, in the present paper, we first handle the sublinear case, and hence make the following assumptions:

$\left(\mathrm{V}_{1}\right) \quad V(x) \in C\left(\mathbb{R}^{3}, \mathbb{R}\right)$ satisfies $\inf _{x \in \mathbb{R}^{3}} V(x)=a_{1}>0$;

$\left(\mathrm{V}_{2}\right)$ for any $M>0$, meas $\left\{x \in \mathbb{R}^{3}: V(x)<M\right\}<+\infty$, where meas denotes the Lebesgue measures;

(K) $K(x) \in L^{2}\left(\mathbb{R}^{3}\right) \cup L^{\infty}\left(\mathbb{R}^{3}\right)$ and $K(x) \geq 0$ for all $x \in \mathbb{R}^{3}$;

(F) there exist constants $\sigma, \gamma \in(1,2)$ and functions $A \in L^{2 /(2-\sigma)}\left(\mathbb{R}^{3}, \mathbb{R}^{+}\right), B \in L^{2 /(2-\gamma)}\left(\mathbb{R}^{3}\right.$, $\mathbb{R}^{+}$) such that $|f(x, u)| \leq A(x)|u|^{\sigma-1}+B(x)|u|^{\gamma-1}, \forall(x, u) \in\left(\mathbb{R}^{3}, \mathbb{R}\right)$;

(G) $g(x) \in L^{2}\left(\mathbb{R}^{3}\right)$ and $g(x) \geq 0$ for a.e. $x \in \mathbb{R}^{3}$.

Before stating our main result, we give several notations. Let $H^{1}\left(\mathbb{R}^{3}\right)$ be the usual Sobolev space endowed with the standard scalar and norm

$$
(u, v)=\int_{\mathbb{R}^{3}}(\nabla u \nabla v+u v) d x ; \quad\|u\|^{2}=\int_{\mathbb{R}^{3}}\left(|\nabla u|^{2}+|u|^{2}\right) d x .
$$


$D^{1,2}\left(\mathbb{R}^{3}\right)$ is the completion of $C_{0}^{\infty}\left(\mathbb{R}^{3}\right)$ with respect to the norm

$$
\|u\|_{D}^{2}:=\|u\|_{D^{1,2}\left(\mathbb{R}^{3}\right)}^{2}=\int_{\mathbb{R}^{3}}|\nabla u|^{2} d x .
$$

Let

$$
E:=\left\{u \in H^{1}\left(\mathbb{R}^{3}\right): \int_{\mathbb{R}^{3}}\left(|\nabla u|^{2}+V(x) u^{2}\right) d x<\infty\right\} .
$$

Then $E$ is a Hilbert space with the inner product

$$
(u, v)_{E}=\int_{\mathbb{R}^{3}}(\nabla u \cdot \nabla v+V(x) u v) d x
$$

and the norm $\|u\|_{E}=(u, u)_{E}^{1 / 2}$. Obviously, the embedding $E \hookrightarrow L^{s}\left(\mathbb{R}^{3}\right)$ is continuous for any $s \in\left[2,2^{*}\right]$. The norm on $L^{s}=L^{s}\left(\mathbb{R}^{3}\right)$ with $1<s<\infty$ is given by $|u|_{s}^{s}=\int_{\mathbb{R}^{3}}|u|^{s} d x$.

Throughout this paper, the letters $C_{i}, d_{i}$ will be used to denote various positive constants which may vary from line to line and are not essential to the problem. We denote by ' - ' weak convergence and by ' $\rightarrow$ ' strong convergence. Also if we take a subsequence of a sequence $\left\{u_{n}\right\}$, we shall denote it again $\left\{u_{n}\right\}$. We use $o(1)$ to denote any quantity which tends to zero when $n \rightarrow \infty$.

Now we state our main result.

Theorem 1.1 (Sublinear) Assume that $\left(\mathrm{V}_{1}\right),\left(\mathrm{V}_{2}\right),(\mathrm{K}),(\mathrm{F})$, and $(\mathrm{G})$ are satisfied. Then problem (1.1) possesses at least one nontrivial solution.

Remark 1 It is not difficult to see that a function $f$ satisfies our assumption (F). For example, let

$$
f(x, u)= \begin{cases}|x| e^{-|x|^{2}}\left[\sigma|u|^{\sigma-2} u \sin ^{2}\left(\frac{1}{|u|^{\varrho}}\right)-\varrho|u|^{\sigma-\varrho-2} \sin \left(\frac{2}{|u|^{\varrho}}\right)\right], & t \neq 0, \\ 0, & t=0,\end{cases}
$$

where $\varrho>0$ small enough and $\sigma \in(1+\varrho, 2)$.

Remark 2 To the best of our knowledge, it seems that Theorem 1.1 is the first result about the existence of solutions for the nonhomogeneous Schrödinger-Poisson equations with sublinear case.

In the second part of this paper, we deal with the following nonhomogeneous Schrödinger-Poisson system:

$$
\begin{cases}-\Delta u+V(x) u+\phi(x) u=k(x)|u|^{q-2} u-h(x)|u|^{p-2} u+g(x), & x \in \mathbb{R}^{3}, \\ -\Delta \phi=u^{2}, \quad \lim _{|x| \rightarrow+\infty} \phi(x)=0, & x \in \mathbb{R}^{3}\end{cases}
$$

where $1<q<2<p<4$, i.e. the nonlinearity of this problem may involve a combination of concave and convex terms.

We assume that $k(x)$ and $h(x)$ are measurable functions satisfying the following conditions: 
(k) $k(x) \in L^{6 /(6-q)}\left(\mathbb{R}^{3}\right) \cap L^{\infty}\left(\mathbb{R}^{3}\right)$ and $k(x) \geq 0$ is not identically zero for a.e. $x \in \mathbb{R}^{3}$;

(h) $h(x) \in L^{\infty}\left(\mathbb{R}^{3}\right)$ and $h(x)>0$ for a.e. $x \in \mathbb{R}^{3}$.

Theorem 1.2 Let $1<q<2<p<4,\left(\mathrm{~V}_{1}\right),\left(\mathrm{V}_{2}\right),(\mathrm{k})$, (h), and $(G)$ hold, then problem (1.2) admits at least one nontrivial solution.

Remark 3 The condition in $\left(\mathrm{V}_{2}\right)$, which implies the compactness of embedding of the working space $E$ and contains the coercivity condition, $V(x) \rightarrow \infty$ as $|x| \rightarrow \infty$, is first introduced by Bartsch and Wang in [40] to overcome the lack of compactness. We are not sure whether Theorem 1.2 holds without the condition $\left(\mathrm{V}_{2}\right)$.

Remark 4 In [23], the author obtains the existence of multiple radially symmetric solutions on $\mathbb{R}^{3}$ for (1.2). In our Theorem 1.2, we do not need the radially symmetric on the potential $V$, so we get the non-radially symmetrical solution for system (1.2) with the concave and convex nonlinearities.

Remark 5 In order to get our results, we have to solve some difficulties. The main difficulty is the loss of compactness of the Sobolev embedding $H^{1}\left(\mathbb{R}^{3}\right)$ into $L^{s}\left(\mathbb{R}^{3}\right), s \in[2,6]$, since this problem is set on $\mathbb{R}^{3}$. To recover the difficulty, some references use the radially symmetric function space, which possesses a compact embedding; see [41]. In our paper, we have the integrability of $k$ and the assumption $1<q<2$ to ensure the space $E$ is compactly embedding in the weighted Lebesgue space (see the following Lemma 2.1).

Remark 6 In [42], the authors first get two solutions for the nonhomogeneous Schrödinger-Poisson equations on $\mathbb{R}^{3}$ with $1<q<2,4<p<6$. Theorem 1.2 can be regarded as complementary work to Wang and Ma (2015) [42].

\section{Nonlinear Schrödinger-Poisson equations with sublinear case}

In this section, we consider the following nonhomogeneous Schrödinger-Poisson system:

$$
\begin{cases}-\Delta u+V(x) u+K(x) \phi(x) u=f(x, u)+g(x), & x \in \mathbb{R}^{3}, \\ -\Delta \phi=K(x) u^{2}, & x \in \mathbb{R}^{3},\end{cases}
$$

where $g \in L^{2}\left(\mathbb{R}^{3}\right), g \not \equiv 0, V$ satisfies $\left(\mathrm{V}_{1}\right)-\left(\mathrm{V}_{2}\right)$, and $f$ satisfies $(\mathrm{F})$.

In Section 1 , we know that the embedding $E \hookrightarrow L^{s}\left(\mathbb{R}^{3}\right)$ is continuous, for any $s \in\left[2,2^{*}\right]$. Furthermore, we have the following result.

Lemma 2.1 ([43], Lemma 3.4) Under assumption $\left(\mathrm{V}_{1}\right)$ and $\left(\mathrm{V}_{2}\right)$, the embedding $E \hookrightarrow$ $L^{s}\left(\mathbb{R}^{3}\right)$ is compact for any $s \in\left[2,2^{*}\right)$.

By Lemma 2.1, there exists $d_{s}>0$ such that

$$
|u|_{s} \leq d_{s}\|u\|_{E}, \quad \forall u \in E
$$

It is well known that problem (2.1) can be reduced to a single equation with a nonlocal term; see [11]. In fact, for every $u \in E$, the Lax-Milgram theorem implies that there exists 
a unique $\phi_{u} \in D^{1,2}\left(\mathbb{R}^{3}\right)$ such that

$$
-\Delta \phi_{u}=K(x) u^{2}
$$

with

$$
\phi_{u}(x)=\frac{1}{4 \pi} \int_{\mathbb{R}^{3}} \frac{K(y) u^{2}(y)}{|x-y|} d y .
$$

If $K \in L^{\infty}\left(\mathbb{R}^{3}\right)$, by (2.3), the Hölder inequality, and the Sobolev inequality, we get

$$
\left\|\phi_{u}\right\|_{D}^{2}=\int_{\mathbb{R}^{3}} K(x) \phi_{u} u^{2} d x \leq \bar{S}^{-2} d_{12 / 5}^{4}|K|_{\infty}^{2}\|u\|_{E}^{4} .
$$

Similarly, if $K \in L^{2}\left(\mathbb{R}^{3}\right)$,

$$
\left\|\phi_{u}\right\|_{D}^{2}=\int_{\mathbb{R}^{3}} K(x) \phi_{u} u^{2} d x \leq \bar{S}^{-2} d_{6}^{4}|K|_{2}^{2}\|u\|_{E}^{4} .
$$

Thus, there exists $C_{0}>0$ such that

$$
\left\|\phi_{u}\right\|_{D}^{2}=\int_{\mathbb{R}^{3}} K(x) \phi_{u} u^{2} d x \leq C_{0}\|u\|_{E}^{4}, \quad \forall K \in L^{2}\left(\mathbb{R}^{3}\right) \cup L^{\infty}\left(\mathbb{R}^{3}\right) .
$$

Now we consider the functional $I$ on $\left(E,\|\cdot\|_{E}\right)$ :

$$
I(u)=\frac{1}{2} \int_{\mathbb{R}^{3}}\left(|\nabla u|^{2}+V(x) u^{2}\right) d x+\frac{1}{4} \int_{\mathbb{R}^{3}} K(x) \phi_{u} u^{2} d x-\varphi(u)-\int_{\mathbb{R}^{3}} g(x) u d x,
$$

where $\varphi(u)=\int_{\mathbb{R}^{3}} F(x, u) d x$.

It follows from $(\mathrm{F})$ that

$$
|F(x, u)| \leq A(x)|u|^{\sigma}+B(x)|u|^{\gamma}, \quad \forall(x, u) \in \mathbb{R}^{3} \times \mathbb{R}
$$

by which, together with (2.4) and the Hölder inequality, we have

$$
\begin{aligned}
\int_{\mathbb{R}^{3}} F(x, u) d x & \leq \int_{\mathbb{R}^{3}}\left(A(x)|u|^{\sigma}+B(x)|u|^{\gamma}\right) d x \\
& \leq|A|_{\frac{2}{2-\sigma}}|u|_{2}^{\sigma}+|B|_{\frac{2}{2-\gamma}}|u|_{2}^{\gamma} \\
& \leq|A|_{\frac{2}{2-\sigma}} d_{2}^{\sigma}\|u\|_{E}^{\sigma}+|B|_{\frac{2}{2-\gamma}} d_{2}^{\gamma}\|u\|_{E}^{\gamma} \\
& <+\infty .
\end{aligned}
$$

Therefore, $\varphi$ and $I$ are well defined. In addition, we need the following lemmas.

Lemma 2.2 ([27], Lemma 4.1) Assume that $\left(\mathrm{V}_{1}\right),\left(\mathrm{V}_{2}\right)$, and $(\mathrm{F})$ are satisfied and $u_{n} \rightarrow u$ in E, then

$$
f\left(x, u_{n}\right) \rightarrow f(x, u) \quad \text { in } L^{2}\left(\mathbb{R}^{3}\right) .
$$


Lemma 2.3 ([27], Lemma 4.2) Assume that $\left(\mathrm{V}_{2}\right),(\mathrm{K})$, and $(\mathrm{F})$ hold. Then $\varphi \in C^{1}(E, \mathbb{R})$ and $\varphi^{\prime}: E \rightarrow E^{*}$ (the dual space of $E$ ) is compact, and hence $I \in C^{1}(E, \mathbb{R})$,

$$
\begin{aligned}
& \left\langle\varphi^{\prime}(u), v\right\rangle=\int_{\mathbb{R}^{3}} f(x, u) v d x \\
& \left\langle I^{\prime}(u), v\right\rangle=\int_{\mathbb{R}^{3}}\left(\nabla u \nabla v+V(x) u v+K(x) \phi_{u} u v-f(x, u) v-g(x) v\right) d x
\end{aligned}
$$

for all $u, v \in E$. Hence, if $u \in E$ is a critical point of $I$, then $\left(u, \phi_{u}\right) \in E \times D^{1,2}\left(\mathbb{R}^{3}\right)$ is a solution of problem (2.1).

We refer the reader to [3] and [11] for the details.

Now we give a proposition, which will be applied to prove Theorem 1.1. Recall that $I \in$ $C^{1}(E, \mathbb{R})$ is said to satisfy the $(P S)$-condition if any sequence $\left\{u_{j}\right\}_{j \in \mathbb{N}}$ is bounded and $I^{\prime}\left(u_{j}\right) \rightarrow$ 0 as $j \rightarrow+\infty$, possesses a convergent subsequence in $E$.

Proposition 2.4 $([44,45])$ Let $E$ be a real Banach space and $I \in C^{1}(E, \mathbb{R})$ satisfy the (PS)condition. If $I$ is bounded from below, then $c=\inf _{E} I$ is a critical value of $I$.

Lemma 2.5 Under the assumptions of Theorem 1.1, I is bounded from below and satisfies the (PS)-condition.

Proof By (K), (G), and (2.5), it follows that

$$
I(u) \geq \frac{1}{2}\|u\|_{E}^{2}-|A|_{\frac{2}{2-\sigma}} d_{2}^{\sigma}\|u\|_{E}^{\sigma}-|B|_{2-\gamma} d_{2}^{\gamma}\|u\|_{E}^{\gamma}, \quad \forall u \in E .
$$

Noting that $\sigma, \gamma \in(1,2)$, we have

$$
I(u) \rightarrow+\infty \quad \text { as }\|u\|_{E} \rightarrow \infty
$$

Thus $I$ is bounded from below.

Let $\left\{u_{n}\right\} \subset E$ be a $(P S)$-sequence of $I$, i.e. $\left\{I\left(u_{n}\right)\right\}$ is bounded and $I^{\prime}\left(u_{n}\right) \rightarrow 0$ as $n \rightarrow+\infty$. By (2.8), $\left\{u_{n}\right\}$ is bounded, and then $u_{n} \rightarrow u$ in $E$ for some $u \in E$. Recall that

$$
(x y)^{1 / 2}(x+y) \leq x^{2}+y^{2}, \quad \forall x, y \geq 0
$$

By $-\Delta \phi_{u}=K(x) u^{2}$ and the Hölder inequality, we obtain

$$
\begin{aligned}
\int_{\mathbb{R}^{3}} K(x)\left(\phi_{u_{n}} u_{n} u+\phi_{u} u_{n} u\right) d x \\
\leq\left(\int_{\mathbb{R}^{3}} K(x) \phi_{u_{n}} u_{n}^{2} d x\right)^{1 / 2}\left(\int_{\mathbb{R}^{3}} K(x) \phi_{u_{n}} u^{2} d x\right)^{1 / 2} \\
\quad+\left(\int_{\mathbb{R}^{3}} K(x) \phi_{u} u_{n}^{2} d x\right)^{1 / 2}\left(\int_{\mathbb{R}^{3}} K(x) \phi_{u_{n}} u^{2} d x\right)^{1 / 2} \\
=\left(\int_{\mathbb{R}^{3}} \nabla \phi_{u_{n}} \cdot \nabla \phi_{u} d x\right)^{1 / 2}\left(\left\|\phi_{u_{n}}\right\|_{D}+\left\|\phi_{u}\right\|_{D}\right)
\end{aligned}
$$




$$
\begin{aligned}
& \leq\left\|\phi_{u_{n}}\right\|_{D}+\left\|\phi_{u}\right\|_{D} \\
& =\int_{\mathbb{R}^{3}} K(x)\left(\phi_{u_{n}} u_{n}^{2}+\phi_{u} u^{2}\right) d x,
\end{aligned}
$$

which implies that

$$
\int_{\mathbb{R}^{3}} K(x)\left(\phi_{u_{n}} u_{n}-\phi_{u} u\right)\left(u_{n}-u\right) d x \geq 0
$$

Since $u_{n} \rightarrow u$ in $L^{2}\left(\mathbb{R}^{3}\right)$ and $g \in L^{2}\left(\mathbb{R}^{3}\right)$, we obtain $\int_{\mathbb{R}^{3}} g\left(u_{n}-u\right) d x=o(1)$. This with Lemma 2.2 shows that

$$
\begin{aligned}
\left\|u_{n}-u\right\|_{E}^{2}= & \left\langle I^{\prime}\left(u_{n}\right)-I^{\prime}(u), u_{n}-u\right\rangle-\int_{\mathbb{R}^{3}} K(x)\left(\phi_{u_{n}} u_{n}-\phi_{u} u\right)\left(u_{n}-u\right) d x \\
& +\int_{\mathbb{R}^{3}}\left(f\left(x, u_{n}\right)-f(x, u)\right) d x \\
\leq & \left\|I^{\prime}\left(u_{n}\right)\right\|_{E^{*}}\left\|u_{n}-u\right\|_{E}-\left\langle I^{\prime}(u), u_{n}-u\right\rangle \\
& +\left(\int_{\mathbb{R}^{3}}\left|f\left(x, u_{n}\right)-f(x, u)\right|^{2} d x\right)^{1 / 2} \cdot\left|u_{n}-u\right|_{2} \\
\rightarrow & 0 .
\end{aligned}
$$

That is, $u_{n} \rightarrow u$ as $n \rightarrow \infty$. Hence the (PS)-condition holds. The proof is complete.

Proof of Theorem 1.1 Theorem 1.1 holds directly by Lemma 2.5 and Proposition 2.4. The proof is complete.

\section{Nonlinear term involving a combination of concave and convex terms}

In this section, we consider the following nonhomogeneous Schrödinger-Poisson system:

$$
\begin{cases}-\Delta u+V(x) u+\phi(x) u=k(x)|u|^{q-2} u-h(x)|u|^{p-2} u+g(x), & x \in \mathbb{R}^{3}, \\ -\Delta \phi=u^{2}, \quad \lim _{|x| \rightarrow+\infty} \phi(x)=0, & x \in \mathbb{R}^{3},\end{cases}
$$

where $1<q<2<p<4$, i.e. the nonlinearity of this problem may involve a combination of concave and convex terms.

It is known that problem (3.1) can be reduced to a single equation see [11]. In fact, for every $u \in E$, the Lax-Milgram theorem implies that there exists a unique $\phi_{u} \in D^{1,2}\left(\mathbb{R}^{3}\right)$ such that

$$
-\Delta \phi_{u}=u^{2}, \quad u \in \mathbb{R}^{3},
$$

with

$$
\phi_{u}(x)=\frac{1}{4 \pi} \int_{\mathbb{R}^{3}} \frac{u^{2}(y)}{|x-y|} d y .
$$

By (3.2), the Hölder inequality, and the Sobolev inequality, we get

$$
\int_{\mathbb{R}^{3}}\left|\nabla \phi_{u}\right|^{2} d x=\int_{\mathbb{R}^{3}} \phi_{u} u^{2} d x \leq|u|_{12 / 5}^{2}\left\|\phi_{u}\right\|_{6} \leq C|u|_{12 / 5}^{2}\left\|\phi_{u}\right\|_{D},
$$


then

$$
\left\|\phi_{u}\right\|_{D} \leq C|u|_{12 / 5}^{2}
$$

and

$$
\int_{\mathbb{R}^{3}} \phi_{u} u^{2} d x \leq C|u|_{12 / 5}^{4} \leq C\|u\|_{E}^{4} .
$$

Therefore, problem (3.1) can be reduced to the following equation:

$$
-\Delta u+V(x) u+\phi_{u} u=k(x)|u|^{q-2} u-h(x)|u|^{p-2} u+g(x), \quad x \in \mathbb{R}^{3} .
$$

We introduce the functional $J: E \rightarrow \mathbb{R}$ defined by

$$
\begin{aligned}
J(u)= & \frac{1}{2} \int_{\mathbb{R}^{3}}\left(|\nabla u|^{2}+V(x) u^{2}\right) d x+\frac{1}{4} \int_{\mathbb{R}^{3}} \phi_{u} u^{2} d x-\frac{1}{q} \int_{\mathbb{R}^{3}} k(x)|u|^{q} d x \\
& +\frac{1}{p} \int_{\mathbb{R}^{3}} h(x)|u|^{p} d x-\int_{\mathbb{R}^{3}} g(x) u d x .
\end{aligned}
$$

By (3.3) and the conditions of Theorem 1.2, all the integrals in (3.4) are well defined and in $C^{1}(E, \mathbb{R})$. Now, it is easy to verify that the weak solutions of (3.1) correspond to the critical points of $J: E \rightarrow \mathbb{R}$ with derivative given by

$$
\begin{aligned}
\left\langle J^{\prime}(u), v\right\rangle= & \int_{\mathbb{R}^{3}}\left[\nabla u \nabla v+V(x) u v+\phi_{u} u v-k(x)|u|^{q-2} u v\right. \\
& \left.+h(x)|u|^{p-2} u v-g(x) v\right] d x .
\end{aligned}
$$

Lemma 3.1 Under the assumptions in Theorem 1.2, the functional J is coercive on $E$.

Proof By (k), we have

$$
\int_{\mathbb{R}^{3}} k(x)|u|^{q} d x \leq|k|_{6 / 6-q} S^{-q / 2}\|u\|_{E}^{q}
$$

For $\|u\|_{E}$ large enough, by (3.5) we obtain

$$
\begin{aligned}
J(u) & =\frac{1}{2} \int_{\mathbb{R}^{3}}\left(|\nabla u|^{2}+V(x) u^{2}\right) d x+\frac{1}{4} \int_{\mathbb{R}^{3}} \phi_{u} u^{2} d x-\frac{1}{q} \int_{\mathbb{R}^{3}} k(x)|u|^{q} d x \\
& \geq \frac{1}{2}\|u\|_{E}^{2}-\frac{1}{q}|k|_{6 /(6-q)} S^{-q / 2}\|u\|_{E}^{q}-c|g|_{2}\|u\|_{E} \\
& \geq \frac{1}{2}\|u\|_{E}^{2}-c_{1}\|u\|_{E}^{q}-c|g|_{2}\|u\|_{E} \rightarrow+\infty,
\end{aligned}
$$

since $1<q<2$. The proof is complete.

Lemma 3.2 Assume that $\left(\mathrm{V}_{1}\right),\left(\mathrm{V}_{2}\right),(\mathrm{k}),(\mathrm{h}),(\mathrm{G})$ hold, and $\left\{u_{n}\right\} \subset E$ is a bounded (PS)sequence of $J$, then $\left\{u_{n}\right\}$ has a strongly convergent subsequence in $E$. 
Proof Consider a sequence $\left\{u_{n}\right\}$ in $E$ which satisfies

$$
J\left(u_{n}\right) \rightarrow c, \quad J^{\prime}\left(u_{n}\right) \rightarrow 0, \quad \sup _{n}\left\|u_{n}\right\|_{E}<+\infty .
$$

Going if necessary to a subsequence, we can assume that $u_{n} \rightarrow u$ in $E$. In view of Lemma $2.1, u_{n} \rightarrow u$ in $L^{s}\left(\mathbb{R}^{3}\right)$ for any $s \in\left[2,2^{*}\right)$. By the derivative of $J$, we easily obtain

$$
\begin{aligned}
\left\|u_{n}-u\right\|_{E}^{2}= & \left\langle J^{\prime}\left(u_{n}\right)-J^{\prime}(u), u_{n}-u\right\rangle+\int_{\mathbb{R}^{3}} k(x)\left(\left|u_{n}\right|^{q-1}-|u|^{q-1}\right)\left(u_{n}-u\right) d x \\
& -\int_{\mathbb{R}^{3}} h(x)\left(\left|u_{n}\right|^{p-1}-|u|^{p-1}\right)\left(u_{n}-u\right) d x-\int_{\mathbb{R}^{3}}\left(\phi_{u_{n}} u_{n}-\phi_{u} u\right)\left(u_{n}-u\right) d x .
\end{aligned}
$$

It is clear that

$$
\left\langle J^{\prime}\left(u_{n}\right)-J^{\prime}(u), u_{n}-u\right\rangle \rightarrow 0 \quad \text { as } n \rightarrow \infty .
$$

By the Hölder inequality and the Sobolev inequality, we have

$$
\begin{aligned}
\left|\int_{\mathbb{R}^{3}} \phi_{u_{n}} u_{n}\left(u_{n}-u\right) d x\right| & \leq\left|\phi_{u_{n}}\right|_{6}\left|u_{n}\right|_{12 / 5}\left|u_{n}-u\right|_{12 / 5} \\
& \leq C_{1}\left\|\phi_{u_{n}}\right\|_{D}\left|u_{n}\right|_{12 / 5}\left|u_{n}-u\right|_{12 / 5} \\
& \leq C_{2}\left|u_{n}\right|_{12 / 5}^{3}\left|u_{n}-u\right|_{12 / 5} \rightarrow 0,
\end{aligned}
$$

since $u_{n} \rightarrow u$ in $L^{s}\left(\mathbb{R}^{3}\right)$ for any $s \in\left[2,2^{*}\right)$. We obtain

$$
\int_{\mathbb{R}^{3}} \phi_{u_{n}} u_{n}\left(u_{n}-u\right) d x \rightarrow 0 \quad \text { as } n \rightarrow \infty .
$$

Similarly we can also obtain

$$
\int_{\mathbb{R}^{3}} \phi_{u} u\left(u_{n}-u\right) d x \rightarrow 0 \quad \text { as } n \rightarrow \infty .
$$

By $2<p<4$, (h), and the Hölder inequality, one has

$$
\begin{aligned}
\left|\int_{\mathbb{R}^{3}} h(x)\left(\left|u_{n}\right|^{p-1}-|u|^{p-1}\right)\left(u_{n}-u\right) d x\right| & \leq|h|_{\infty}\left(\left|u_{n}\right|_{p}^{p-1}+|u|_{p}^{p-1}\right)\left|u_{n}-u\right|_{p} \\
& \rightarrow 0 \quad \text { as } n \rightarrow \infty
\end{aligned}
$$

By $1<q<2$, (k), and the Hölder inequality, one has

$$
\begin{aligned}
& \int_{\mathbb{R}^{3}} k(x)\left|u_{n}\right|^{q-1}\left(u_{n}-u\right) d x \\
& \quad=\int_{\mathbb{R}^{3}} k(x)^{\frac{q-1}{q}} k(x)^{\frac{1}{q}}\left|u_{n}\right|^{q-1}\left(u_{n}-u\right) d x \\
& \quad \leq|k|_{\infty}^{1-\frac{1}{q}}\left[\int_{\mathbb{R}^{3}}\left(k(x)^{\frac{1}{q}}\left|u_{n}\right|^{q-1}\right)^{\frac{6}{6-q}} d x\right]^{\frac{6-q}{6}}\left(\int_{\mathbb{R}^{3}}\left(u_{n}-u\right)^{\frac{6}{q}} d x\right)^{\frac{q}{6}}
\end{aligned}
$$




$$
\begin{aligned}
& \leq|k|_{\infty}^{1-\frac{1}{q}}\left(\int_{\mathbb{R}^{3}} k(x)^{\frac{6}{6-q}} d x\right)^{\frac{6-q}{6} \cdot \frac{1}{q}}\left(\int_{\mathbb{R}^{3}}\left|u_{n}\right|^{\frac{6 q}{6-q}} d x\right)^{\frac{6-q}{6 q}(q-1)}\left|u_{n}-u\right|_{\frac{6}{q}} \\
& =|k|_{\infty}^{1-\frac{1}{q}}|k|_{\frac{6}{6-q}}^{\frac{1}{q}}\left|u_{n}\right|_{\frac{6 q}{6-q}}^{q-1}\left|u_{n}-u\right|_{\frac{6}{q}} \rightarrow 0 \quad \text { as } n \rightarrow \infty,
\end{aligned}
$$

since $3<\frac{6}{q}<6, u_{n} \rightarrow u$ in $L^{s}\left(\mathbb{R}^{3}\right)$ for any $s \in\left[2,2^{*}\right)$.

Similarly, we also obtain

$$
\int_{\mathbb{R}^{3}} k(x)|u|^{q-1}\left(u_{n}-u\right) d x \rightarrow 0 \quad \text { as } n \rightarrow \infty
$$

Therefore, by (3.7)-(3.11), we get $\left\|u_{n}-u\right\|_{E} \rightarrow 0$. The proof is complete.

Proof of Theorem 1.2 In view of Proposition 2.4 and Lemma 3.2, we only need to check that $\left\{u_{n}\right\}$ is bounded in $E$,

$$
\begin{aligned}
\tilde{c}_{0}+1+\|u\|_{E} \geq & J\left(u_{n}\right)-\frac{1}{4}\left\langle J^{\prime}\left(u_{n}\right), u_{n}\right\rangle \\
= & \frac{1}{4}\left\|u_{n}\right\|_{E}^{2}+\left(\frac{1}{4}-\frac{1}{q}\right) \int_{\mathbb{R}^{3}} k(x)\left|u_{n}\right|^{q} d x+\left(\frac{1}{p}-\frac{1}{4}\right) \int_{\mathbb{R}^{3}} h(x)\left|u_{n}\right|^{p} d x \\
& -\frac{3}{4} \int_{\mathbb{R}^{3}} g(x) u_{n} d x \\
\geq & \frac{1}{4}\left\|u_{n}\right\|_{E}^{2}+\left(\frac{1}{4}-\frac{1}{q}\right)|k|_{6 /(6-q)} S^{-q / 2}\|u\|_{E}^{q}-\frac{3}{4}|g|_{2}\left|u_{n}\right|_{2}
\end{aligned}
$$

for $n$ large enough. Since $g \in L^{2}\left(\mathbb{R}^{3}\right)$, it follows from $1<q<2$ that $\left\{u_{n}\right\}$ is bounded in $E$. The proof is complete.

\section{Competing interests}

The authors declare that they have no competing interests.

\section{Authors' contributions}

The main idea of this paper was proposed by SM. LW and XW performed all the steps of the proofs in this research. All authors read and approved the final manuscript.

\section{Author details}

'School of Sciences, Tianjin Chengjian University, Tianjin, 300384, China. ${ }^{2}$ School of Mathematical Sciences and LPMC, Nankai University, Tianjin, 300071, China. ${ }^{3}$ School of Mathematics and Computer Science, Shangrao Normal University, Shangrao, Jiangxi 334001, China.

\section{Acknowledgements}

L Wang is partially supported by Tian Yuan Special Foundation (No. 11526148). S Ma is partially supported by NSFC (No. 11571187). X Wang is partially supported by NSFC (No. 11461056).

Received: 15 December 2015 Accepted: 4 April 2016 Published online: 07 April 2016

\section{References}

1. Benguria, R, Bris, H, Lieb, E: The Thomas-Fermi-von Weizsäcker theory of atoms and molecules. Commun. Math. Phys. 79, $167-180(1981)$

2. Lieb, EH: Thomas-Fermi and related theories and molecules. Rev. Mod. Phys. 53, 603-641 (1981)

3. Benci, V, Fortunato, D: An eigenvalue problem for the Schrödinger-Maxwell equations. Topol. Methods Nonlinear Anal. 11, 283-293 (1998)

4. Lions, PL: Solutions of Hartree-Fock equations for Coulomb systems. Commun. Math. Phys. 109, 33-97 (1984)

5. Markowich, P, Ringhofer, C, Schmeiser, C: Semiconductor Equations. Springer, New York (1990)

6. Ruiz, D: The Schrödinger-Poisson equation under the effect of a nonlinear local term. J. Funct. Anal. 237, 655-674 (2006)

7. Seok, J: On nonlinear Schrödinger-Poisson equations with general potentials. J. Math. Anal. Appl. 401, 672-681 (2013) 
8. Ambrosetti, A, Ruiz, D: Multiple bound states for the Schrödinger-Poisson equation. Commun. Contemp. Math. 10, 391-404 (2008)

9. Azzollini, A, Pomponio, A: Ground state solutions for the nonlinear Schrödinger-Maxwell equations. J. Math. Anal. Appl. 345, 90-108 (2008)

10. Cerami, G, Vaira, G: Positive solution for some non-autonomous Schrödinger-Poisson systems. J. Differ. Equ. 248 521-543 (2010)

11. D'Aprile, T, Mugnai, D: Solitary waves for nonlinear Klein-Gordon-Maxwell and Schrödinger-Maxwell equations. Proc. R. Soc. Edinb., Sect. A 134, 893-906 (2004)

12. Huang, LR, Rocha, EM, Chen, JQ: Two positive solutions of a class of Schrödinger-Poisson system with indefinite nonlinearity. J. Differ. Equ. 255, 2463-2483 (2013)

13. Sun, JT: Infinitely many solutions for a class of sublinear Schrödinger-Maxwell equations. J. Math. Anal. Appl. 390, 514-522 (2012)

14. Sun, JT, Wu, TF: On the nonlinear Schrödinger-Poisson system with sign-changing potential. Z. Angew. Math. Phys. (2015). doi:10.1007/s00033-015-0494-1

15. Wang, J, Tian, LX, Xu, JX, Zhang, FB: Existence and concentration of positive solutions for semilinear Schrödinger-Poisson systems in $\mathbb{R}^{3}$. Calc. Var. 48, 243-273 (2013)

16. Zhao, LG, Zhao, FK: Positive solutions for Schrödinger-Poisson equations with a critical exponent. Nonlinear Anal. 70, 2150-2164 (2009)

17. Ambrosetti, A: On Schrödinger-Poisson systems. Milan J. Math. 76, 257-274 (2008)

18. Coclite, GM: A multiplicity result for the nonlinear Schrödinger-Maxwell equations. Commun. Appl. Anal. 7(2-3), 417-423 (2003)

19. Coclite, GM: A multiplicity result for the Schrödinger-Maxwell equations with negative potential. Ann. Pol. Math 79(1), 21-30 (2002)

20. D'Aprile, T, Mugnai, D: Non-existence results for the coupled Klein-Gordon-Maxwell equations. Adv. Nonlinear Stud. 4(3), 307-322 (2004)

21. Kikuchi, H: On the existence of a solution for elliptic system related to the Maxwell-Schrödinger equations. Nonlinear Anal. 67(5), 1445-1456 (2007)

22. Mercuri, C: Positive solutions of nonlinear Schrödinger-Poisson systems with radial potentials vanishing at infinity. Atti Accad. Naz. Lincei Cl. Sci. Fis. Mat. Natur. Rend. Lincei (9) Mat. Appl. 19(3), 211-227 (2008) (English summary)

23. Salvatore, A: Multiple solitary waves for a non-homogeneous Schrödinger-Maxwell system in $\mathbb{R}^{3}$. Adv. Nonlinear Stud. 6(2), 157-169 (2006)

24. Wang, ZP, Zhou, HS: Positive solutions for a nonlinear stationary Schrödinger-Poisson system in $\mathbb{R}^{3}$. Discrete Contin. Dyn. Syst. 18, 809-816 (2007)

25. Zhao, LG, Zhao, FK: On the existence of solutions for the Schrödinger-Poisson equations. J. Math. Anal. Appl. 346(1), 155-169 (2008)

26. Sun, JT, Chen, HB, Nieto, JJ: On ground state solutions for some non-autonomous Schrödinger-Poisson systems. J. Differ. Equ. 252, 3365-3380 (2012)

27. Ye, YW, Tang, CL: Existence and multiplicity of solutions for Schrödinger-Poisson equations with sign-changing potential. Calc. Var. 53, 383-411 (2015)

28. Wu, TF: Multiplicity results for a semi-linear elliptic equation involving sign-changing weigh function. Rocky Mt. J. Math. 39(3), 995-1011 (2009)

29. Huang, LR, Rocha, EM: A positive solution of a Schrödinger-Poisson system with critical exponent. Commun. Math. Anal. 15, 29-43 (2013)

30. Huang, LR, Rocha, EM, Chen, JQ: Positive and sign-changing solutions of a Schrödinger-Poisson system involving a critical nonlinearity. J. Math. Anal. Appl. 408, 55-69 (2013)

31. Shen, ZP, Han, ZQ: Multiple solutions for a class of Schrödinger-Poisson systems with indefinite nonlinearity. J. Math. Anal. Appl. (2015). doi:10.1016/j.jmaa.2015.01.071

32. Sun, MZ, Su, JB, Zhao, LG: Infinitely many solutions for a Schrödinger-Poisson system with concave and convex nonlinearities. Discrete Contin. Dyn. Syst. 35, 427-440 (2015)

33. Wu, TF: Four positive solutions for a semilinear elliptic equation involving concave and convex nonlinearities. Nonlinear Anal. 70, 1377-1392 (2009)

34. Wu, TF: The Nehari manifold for a semilinear elliptic system involving sign-changing weight functions. Nonlinear Anal. 68, 1733-1745 (2008)

35. Chen, SJ, Tang, CL: Multiple solutions for a non-homogeneous Schrödinger-Maxwell and Klein-Gordon-Maxwell equations on $\mathbb{R}^{3}$. Nonlinear Differ. Equ. Appl. 17, 559-574 (2010)

36. Ding, L, Li, L, Zhang, JL: Multiple solutions for nonhomogeneous Schrödinger-Poisson system with asymptotical nonlinearity in $\mathbb{R}^{3}$. Taiwan. J. Math. 17(5), 1627-1650 (2013)

37. Du, M, Zhang, FB: Existence of positive solutions for a nonhomogeneous Schrödinger-Poisson system in $\mathbb{R}^{3}$. Int. J. Nonlinear Sci. 16(2), 185-192 (2013)

38. Jiang, YS, Wang, ZP, Zhou, HS: Multiple solutions for a nonhomogeneous Schrödinger-Maxwell system in $\mathbb{R}^{3}$. Nonlinear Anal. 83, 50-57 (2013)

39. Yang, MB, Li, BR: Solitary waves for non-homogeneous Schrödinger-Maxwell system. Appl. Math. Comput. 215, 66-70 (2009)

40. Bartsch, T, Wang, ZQ: Existence and multiplicity results for some superlinear elliptic problem on $\mathbb{R}^{N}$. Commun. Partial Differ. Equ. 20, 1725-1741 (1995)

41. Strauss, WA: Existence of solitary waves in higher dimensions. Commun. Math. Phys. 55, 149-162 (1977)

42. Wang, LX, Ma, SW: Multiple solutions for a nonhomogeneous Schrödinger-Poisson system with concave and convex nonlinearity. Acta Math. Appl. Sin., Engl. Ser. (submitted)

43. Zou, WM, Schechter, M: Critical Point Theory and Its Applications. Springer, New York (2006)

44. Mawhin, J, Willem, M: Critical Point Theory and Hamiltonian Systems. Springer, New York (1989)

45. Willem, M: Minimax Theorems. Birkhäuser, Boston (1996) 